\title{
Research on Navigation and Positioning Technology of Intelligent Vehicle Based on GNSS/INS Integrated Navigation System
}

\author{
Tong $\operatorname{Jin}^{1}$, Yuansheng Liu ${ }^{2, *}$, Wenjuan Zhang ${ }^{1}$, Jiansuo Yang ${ }^{1}$ and Xue Wang ${ }^{1}$ \\ ${ }^{1}$ Information College of Beijing Union University, Beijing 100101, China \\ ${ }^{2}$ Beijing Key Laboratory of Information Service Engineering, Beijing Union University, Beijing 100101, China
}

\begin{abstract}
Intelligent automobile emerged when intelligence became the main requirement of the times.. Aiming at the problem of accurate navigation of intelligent vehicle, an integrated navigation positioning method based on GNSS(Global Navigation Satellite System)/INS(Inertial Navigation System) used to acquire information about vehicle's location and vehicle's function was introduced in this paper. This method can produce a map which is suitable forintelligent vehicle. In the real-time navigation, unmanned driving of intelligent vehicle can be realized through the UTM (Universal Transverse Mercator) algorithm with a priori map to obtain the real-time running track.
\end{abstract}

Keywords: GNSS, INS, UTM, Map for vehicle, Intelligent drive.

\section{INTRODUCTION}

With the development of automotive electronics and intelligent control technology, intelligent vehicle has become a hot research topic in the field of intelligent control. Precision in driving intelligent vehicle can be achieved according to the predetermined route dependent on the precise navigation system in unmanned condition. The current navigation methods include satellite system navigation, guidance magnets or guide cable navigation, inertial navigation system and GNSS/INS integrated navigation system [1]. However, navigation based on satellite system has low accuracy when there is occlusion. The navigation based on guidance magnets or guide cable has poor scalability. The navigation's accuracy based on inertial navigation system is not high. All of these cannot meet the accuracy requirement for driving intelligent vehicle.

In 2011, Kun Liu, in the article of "Application of GNSS/INS Integrated Navigation on Flight Inspection System", pointed out that the integrated navigation system is more accurate and reliable than the individual subsystems. In 2014, in the article of "The Positioning Accuracy Analysis about Integrated GNSS/INS Navigation System", Xiaoyan Wang verified that the precision of GNSS/INS can achieve centimeter level. Moreover, Weifeng Pan successfully applied the GNSS/INS navigation system to the motor vehicle driver intelligent examination system in his study. Therefore, the navigation method based on GNSS/INS integrated position navigation system is precise and stable when intelligent vehicle implements intelligent driving.

*Address correspondence to this author at the Beijing Key Laboratory of Information Service Engineering, Beijing Union University Beijing 100101, China; E-mail: yuansheng@buu.edu.cn
This paper mainly introduced navigation device and technology, and collected map and described real time navigation. A brief introduction about the structure of intelligent vehicle is presented in section 2 . Section 3 summarizes the GNSS/INS integrated navigation device and relevant technology. To realize the precise navigation, collecting a map for vehicle was required, which is introduced in section 4 and section 5 . The real-time navigation is described step by step in section 6 .

\section{THE OVERALL STRUCTURE OF INTELLIGENT VEHICLE}

The overall architecture of intelligent vehicle iscomposed of the perception layer, information fusion layer, decision layer, control layer and vehicle interface (Fig. 1). The perceptual layer consists of the visual perception, the perception of radar and the attitude of the current vehicle position information. Through visual perception and the perception of radar fromthe vehicle's eye, one can clearly understand the surrounding environment immediately. For instance, visual perception can determine traffic lights and the state of traffic lights, while radar can detect how far the obstacles are. But visual perception and the perception of radar cannot determine the terminal point and comprehensive information, therefore, they can only be used to make path planning in local small range. Navigation equipment can not only make the vehicle familiar about the current basic information such as latitude, longitude, speed, heading but also make a priori map as an actual map in the driver's brain. In this way, the vehicle itself determines current information such as current location and road condition which are enough to guide decision-making layer and make correct judgment depend on car's condition to send appropriate instruction to the control layer. 


\begin{tabular}{|l|l|l|l|l|}
\hline $\begin{array}{c}\text { perception } \\
\text { layer }\end{array}$ & Information fusion layer & decision layer & control layer & vehicle interface \\
\hline
\end{tabular}
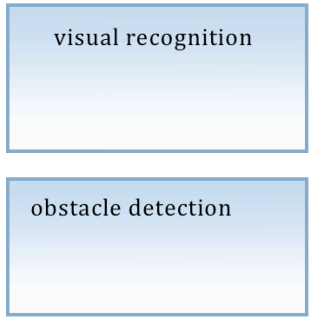

vehicle state

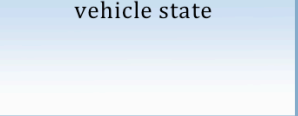

path planning behavioral decision making

Fig. (1). The overall structure of intelligent vehicle.

\section{GNSS/INS INTEGRATED NAVIGATION SYSTEM}

\subsection{The Composition Of GNSS/INS Integrated Naviga- tion System}

GNSS is a space-based radio navigation system which determines information of user's position by measuring signal delay among four satellites whose ephemeris has been known and receiver antenna [2]. INS which is based on the principle of Newtonian mechanics has some navigation parameters like carrier's angle velocity that gyro and accelerometer measure relatively to the inertial space and the three-dimensional velocity, position and attitude information are obtained by integration [3-5].

GNSS/INS integrated navigation can be divided into three levels, namely, loose coupling, tight coupling and deep coupling $[6,7]$. In loose coupling mode, GNSS and INS work as systems independent of each other and provide independent navigation results that are processed by estimator which is generally a Kalman filter to obtain the desired navigation solution.

In this project, tight coupling mode was used. In tight coupling mode, GNSS and INS are no longer used as navigation systems but as simple sensors which determine pseudo range, pseudo range rate, acceleration and angular velocity of rotation and so on. These measurements are processed by estimator like Kalman filter to obtain the optimal navigation solution. Typically, tight coupling system's position result is more accurate than loose coupling system. Moreover, tight coupling system can still work while the system has less than 4 satellites.

The integrated navigation system is composed of base station which contains a receiving antenna, C290 series of receiver and mobile radio station which contains SPANCPT, host antenna, slave antenna mobile station and slave receiver. Base station and mobile radio station should be correctly connected, respectively, while the base station is set up in the place that has no obstacle and mobile radio station is fixed in the object which needs to be positioned.
Navigation equipment will work normally after providing $+12 \mathrm{~V}$ DC power supply to the equipment and initialing two stations.

\subsection{The Advantage Of Integrated Navigation System}

SPAN-CPT is a GNSS/INS tight coupling integrated navigation system which consists of GNSS system, optical fiber gyroscope, quartz accelerometer, DSP(Digital Signal Processing) processor and Kalman filter. SPAN-CPT's available positioning modes include single point, RTK and post processing method. When using single point positioning method, precision of SPAN-CPT is 1.2 meters RMS. Real-time differential and post differential processing have no obvious difference when GNSS's observation is better, and their precision levels follow centimeter level. Post differential processing has an obvious advantage that its precision is still at centimeter level after losing lock 10s when GNSS is the losing lock.

Navigation becomes unreliable or out of commission under the condition that mobile radio station is blocked by trees and buildings thus making it hard for mobile radio station to search enough satellites. Positioning and navigation of inertial navigation system shift with time due to the cumulative error of IMU internal data without external reference conditions. A more powerful navigation system is generated by combining two navigation technologies. Absolute position and speed's precision that GNSS measures can be used for compensating the measurement error of IMU. Result of stable relative position that INS measures is obtained continuously when GNSS's solution is poor or it cannot be used.

The RTK model also improves positioning precision of navigation. RTK (Real Time Kinematic), a real time dynamic measurement technology, is based on the carrier phase observation. Moreover, it is a breakthrough in the development of measurement technology which is composed of a base station's receiver, data link and mobile radio station's 


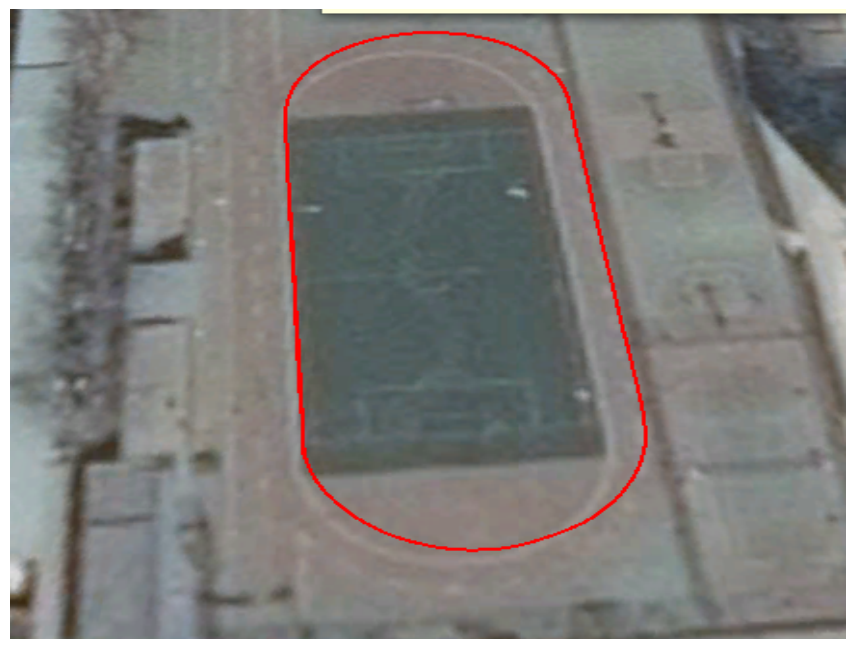

Fig. (2). RTK differential positioning map.

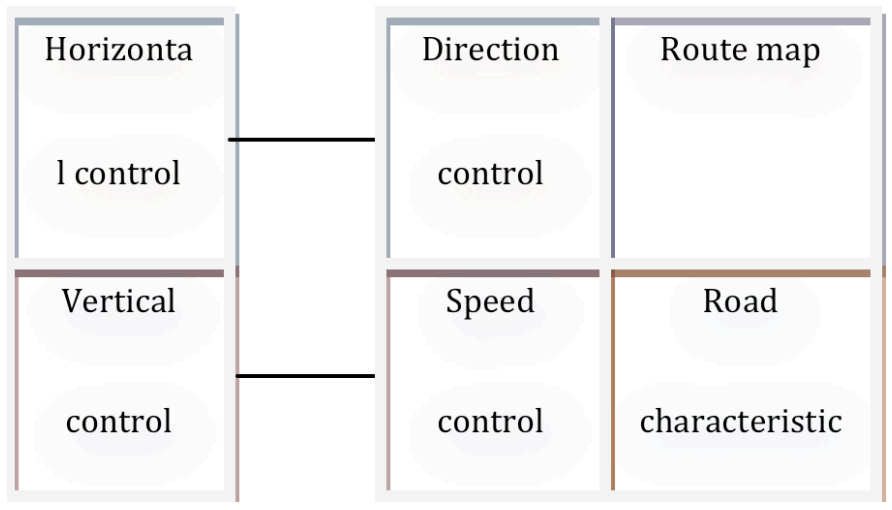

Fig. (3). Vehicle control chart.

receiver. A receiver placed in the base station as a reference station sends measurement data and information to the mobile radio station GPS receiver which receives GPS satellite signal via radio transmission equipment. At the same time, through wireless receiving apparatus mobile radio station's GPS receiver, the data is received that base station sends and real time three-dimension coordinate along with its precision is figured out. Mobile radio station's GPS receiver can figure out plane coordinate $\mathrm{X}, \mathrm{Y}$ and altitude $\mathrm{H}$ of each point of mobile radio station according to coordinate difference $\Delta \mathrm{X}, \Delta \mathrm{Y}, \Delta \mathrm{H}$ between the base station and mobile radio station and each point's WGS-84 coordinate is obtained by reference coordinate.

From the RTK differential positioning map (Fig. 2), it can be seen that there is deviation which is due to "National security plug-in" added in Chinese map when acquisition path is showed in Google earth. At the same time, it is clear that RTK differential positioning in the navigation has a high precision as shown in the figure.

\section{MAP FOR VEHICLE}

Map is of great significance for driver because map can indicate the general direction and determine the specific route. Driver selects the driving road and determines the specific path based on prior map knowledge registered in the driver's brain due to the daily life of driving. The decision of intelligent vehicle should be made based on the principles followed by the driver. The vehicle is made familiar about the road by its own prior map knowledge which is map for the vehicle. Map for the vehicle is the same as common electronic map which indicates the available road. But there is a difference that the map for vehicle can assist decision-making layer to control vehicle's speed. The road characteristics specifically refer to intrinsic attribute of the road such as straight road or curved road. For controlling the vehicle, the control function is divided into horizontal control and vertical control [8]. Horizontal control determines the direction control and the vertical control determines the speed control. Map for vehicle's route serves as the basis of the direction control and map for vehicle's road characteristic value serves as an important reference of speed control. Therefore map for vehicle should be composed of two parts: route map and road characteristic value (Fig. 3).

\subsection{Route Map}

A series of latitude and longitude points are connected into sections and a series of sections are spliced into a route 


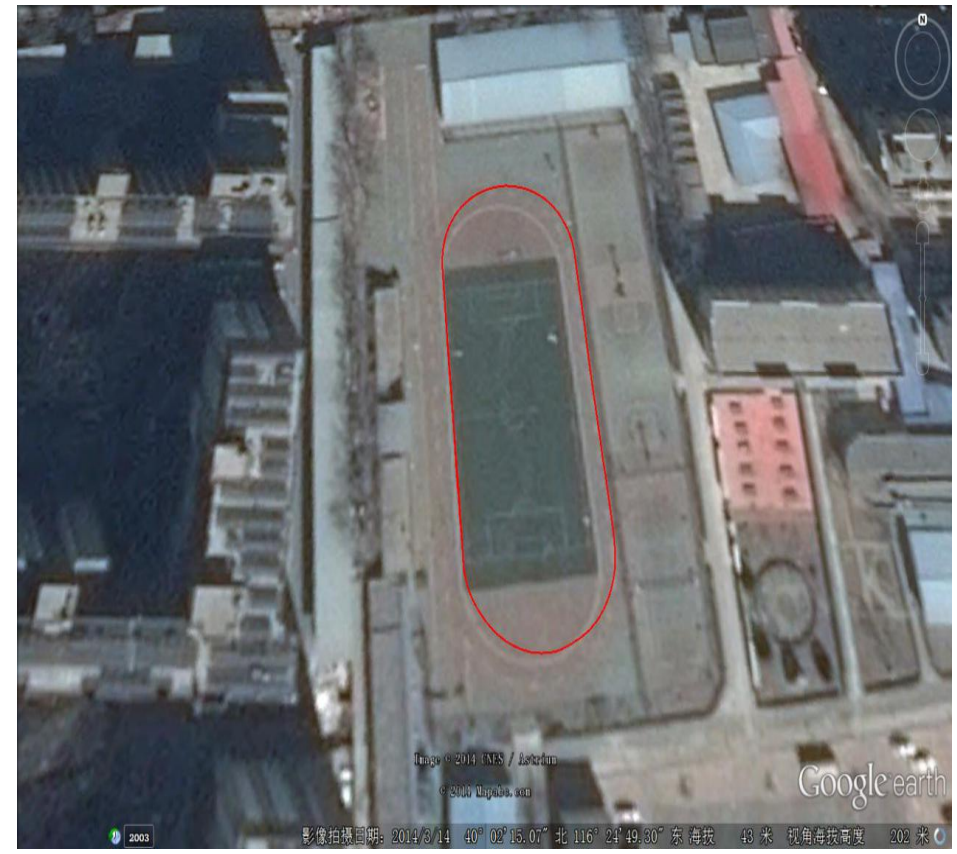

Fig. (4). The acquisition route map.

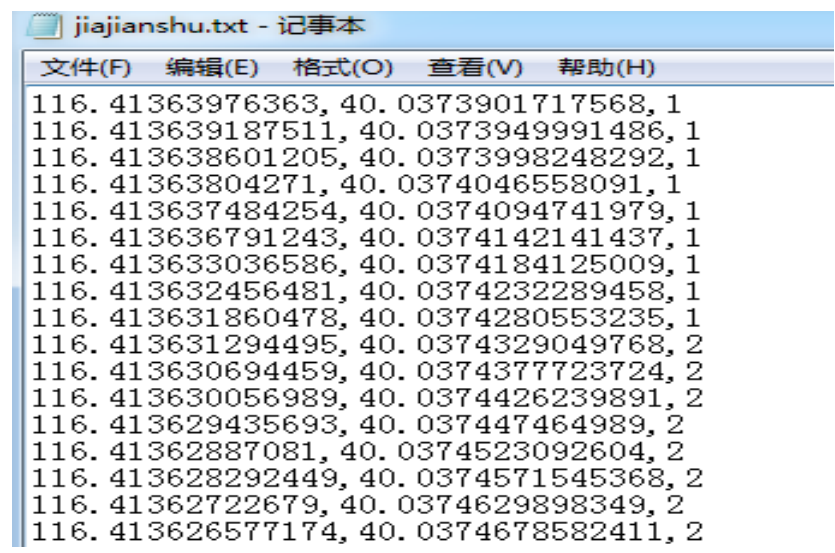

Fig. (5). The result of map for vehicle.

map. Map for vehicle's route map contains many longitude and latitude points. The key of vehicle angle control is extending the trend of those points. The acquisition route (Fig. 5) shows us a simple route map displayed in Google earth.

\subsection{Road Characteristic Value}

There is a big difference between the map for intelligent driving and general map that the map for intelligent driving includes road characteristic value displayed in Table 1. Preliminary, road characteristic value is divided based on the degree of road curve and factors that influence vehicle's speed on the road. Vehicle's speed can be high when the bending degree of road is small and it can be low when the bending degree of road is large. Therefore, labeling road characteristic value is beneficial to control the speed.

\section{COLLECT MAP}

\subsection{Serial Port Receiver}

The navigation device is connected through the USB line to the IPC(Industrial Process Connection). After configuring navigation device and opening the corresponding serial port, navigation device constantly sends data to the IPC. Only two kinds of frames carrying GPS data can be received and other frame's information would be filtered out by processing through software. One is "bestposb" frame sent by a second time, which is used to monitor the reliability of the current GPS signals by examining the number of search satellite and the RTK status flag. The other frame is "inspvasb" frame sent by a second 50 times, which is mainly used to examine the current vehicle's position, speed, heading Angle and related vehicle attitude information. Each frame contains 72 bytes. These 72 bytes are stored in an array of bytes. According to the user manual of the 
Table 1. Road Characteristic Value.

\begin{tabular}{|c|c|}
\hline Road Characteristic Value & Corresponding Description \\
\hline \hline 0 & Start the vehicle \\
\hline 1 & Slow speed \\
\hline 2 & Middle speed \\
\hline 3 & High speed \\
\hline 4 & Sharp turn in the road \\
\hline 5 & Cross in the road \\
\hline 6 & bridge \\
\hline 7 & U turn \\
\hline 8 & Traffic light \\
\hline 9 & Speed limit road \\
\hline 10 & Stop the vehicle \\
\hline
\end{tabular}

navigation device, vehicle's status can be parsed. After obtaining the longitude and latitude data, the data is stored in the "Map" list. During the modeling of the map, the current road section is provided with road properties according to the table of road characteristic value. In view of the current road attribute, decision-making layer can also be modified accordingly to make the intelligent vehicle more intelligent.

Because of fast data transmission from the navigation device and slow driving of the vehicle while designing the map, the two points in the "map" list becomes too close. This would result in the production of huge data. In fact, there is no need to acquire large data .Therefore, UTM projection should be used for filtering out the points that do not meet the condition.

\subsection{Utm Projection}

UTM Projection (Universal Transverse Mercator Projection) is a transverse cut conformal elliptic cylindrical projection. Elliptic cylindrical cutting earth to 80 degrees south latitude, 84 degrees north latitude as two high ring, has no deformation after the projection two is cut through the longitude, and the central meridian length ratio is 0.9996 [9]. The Gauss Kruger projection is similar, with no deformation of the projection angle, the central meridian, linear, and as a symmetry axis projection, scale factor is the central meridian 0.9996 which ensure that there are two undistorted standard meridian from the central meridian about $180 \mathrm{~km}$.

UTM projection in zoning method is 84 degrees north latitude to 80 degrees south latitude and the longitude is divided into 60 zones, each with 6 degrees from 180 degrees west longitude. The data of two standards from the central meridian of longitude is about $180 \mathrm{KM}$, with the central meridian, and the proportion coefficient is 0.9996 .

There is no other way to examine latitude and longitude directly. UTM projection can transfer WGS coordinate system into geodetic coordinate system. Geodetic coordinate system can be processed by comprehending plane geometry.

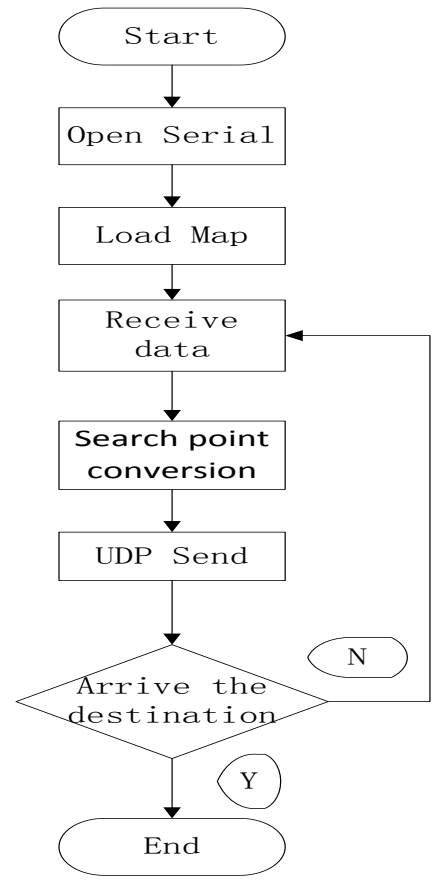

Fig. (6). Flow chart of real time navigation algorithm.

Equation 1 can be used to calculate the distance between two longitude and latitude points.

$d=\sqrt{(\mathrm{x} 1-\mathrm{x} 2)^{2}+(\mathrm{y} 1-\mathrm{y} 2)^{2}}$

If the set distance value is 0.5 meter, the distance $\mathrm{d}$ calculated by the equation is compared to 0.5 . However, if the distance is more than 0.5 , the latter point would be saved otherwise the latter point would be deleted.

\subsection{Save Data}

The data collected are stored in the form of tuple. Each tuple is composed of longitude, latitude and road characteristic value. All data can be stored in the text file or database. In the project, the data were stored in the text file. The text file is equivalent to a map for intelligent vehicle. The map for vehicle was completed by following the steps discussed above. The diagram below shows the format of the map for vehicle.

\section{REAL TIME NAVIGATION ALGORITHM}

\subsection{Flow Chart Of Navigation Algorithm}

Flow chart of navigation algorithm is just like figure (Fig. 6).

\subsection{Search Point Conversion}

Search point conversion is based on the current location of vehicle. In the project, accurate positioning point of vehicle was the front center of the vehicle. According to the current point, points on the map satisfied the condition of dis- 


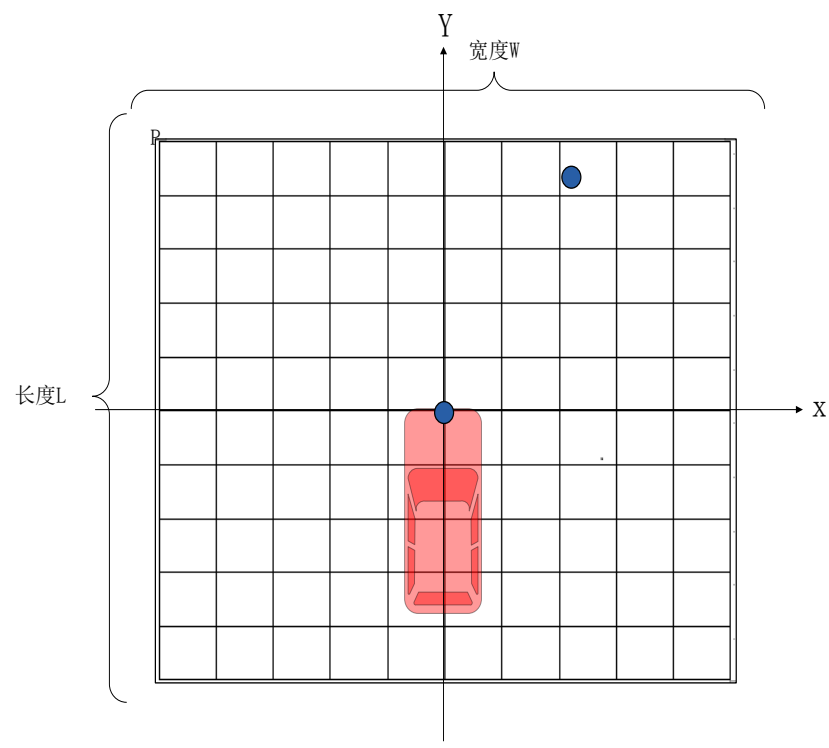

Fig. (7). Body coordinate system.

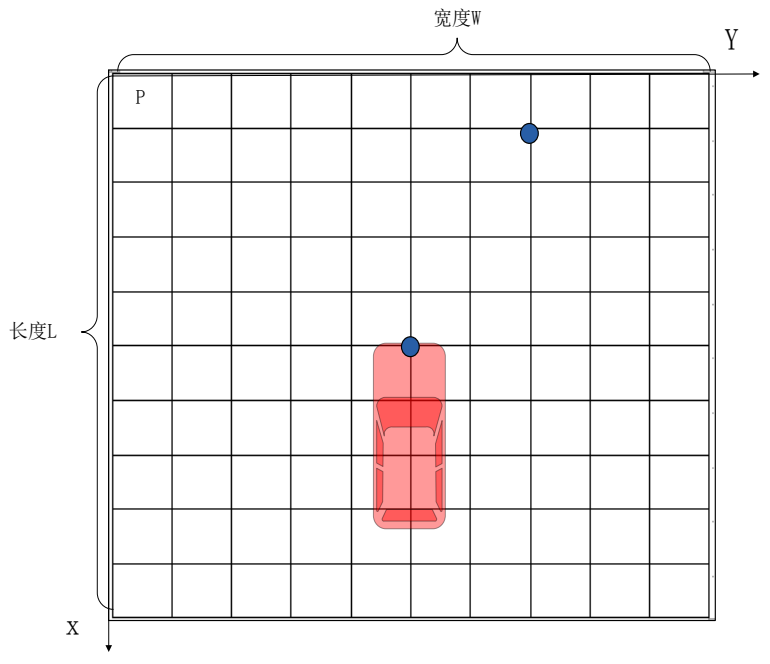

Fig. (8). Grid coordinate system.

tance which was calculated and projected onto the vehicle body coordinate system.

The first step involved the calculation of distance between two latitude and longitude points.

The latitude and longitude of the current position can be converted into coordinate of the geodetic coordinate system by UTM projection. along with the points on the map. In the same Cartesian coordinate system, the distance $d$ between two points could be calculated by using the formula.

The second step involved calculation of the deviation angle $\beta$, the front center of the vehicle and a point of map from a vector. There is an angle called $\alpha$ between the vector and the north. The angle $\alpha$ minus the heading angle Azimuth represents the deviation angle $\beta$.

The third step involved examination of the projection to the body coordinate system.
The point of map is transferred to the body coordinate system with the use of deviation angle and distance. The specific method is as follows. The front is the origin of coordinates and the direction of the head is taken in the positive direction of axis $\mathrm{X}$, and the right direction of the front vertical is taken in the positive direction of axis $y$. The point of the map can be transferred to the body coordinate system (Fig. 7) by the trigonometric transformation formula. At the same time, $\mathrm{x}$ and $\mathrm{y}$ meet two conditions. One is that, $\mathrm{x}$ is greater than -10 and less than 10 . The other one is that $y$ is greater than -30 and less than 30 .

The fourth step involved the offset to the grid coordinate system [10].

Grid coordinate system (Fig. 8) is a $300 * 100$ rectangular grids. Each grid is the small square whose side length is 


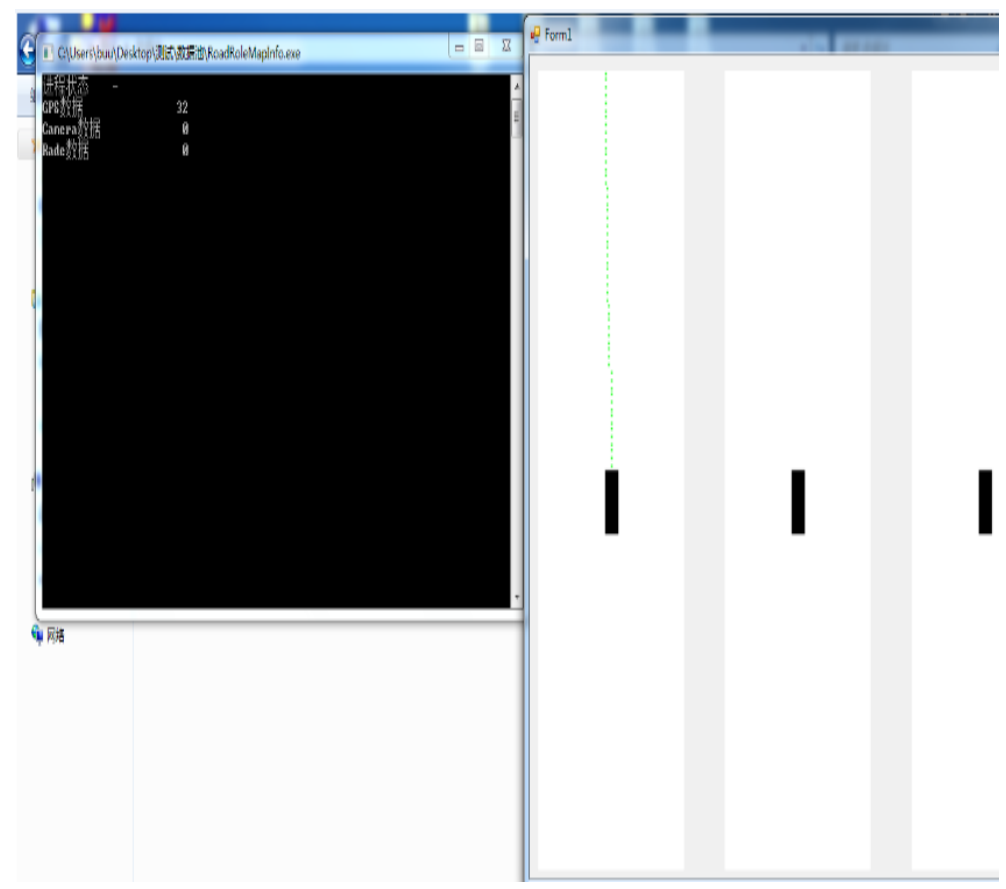

Fig. (9). Guide map.

$20 \mathrm{~cm}$. The point on the left above of the rectangular grids is treated as the origin of the coordinate system $(0,0)$. The coordinate of the front center point is ruled as $(150,50)$. The body coordinate system can be transferred to the grid coordinate system by the following equation (2) and equation (3).

$$
\begin{aligned}
& P o \text { int } \mathrm{x}=150-\mathrm{x} / 0.2 \\
& P o \text { inty }=50+y / 0.2
\end{aligned}
$$

All of the points which meet the search scope in the map are processed like mentioned above.

\subsection{Udp Send}

Coordinate data after conversion is sent to information fusion layer by using the udp protocol. The guide map (Fig. 9) below shows that the data fusion layer receives data transmitted from the navigation group's result. All the points can be seen as a discrete guiding wire to guide the vehicle to move forward.

\section{CONCLUSION}

According to a large number of real vehicle field tests, without considering traffic lights, and obstacles, the vehicle can also be guided to reach the specified destination from the specified starting point without manual intervention. When the state of RTK is not ideal to be maintained for a long time, the navigation accuracy cannot be guaranteed.

The map navigation strategy based on the GNSS/INS integrated navigation system can guide the vehicle to drive accurately along the known path. There is no doubt that multi sensors' information should be considered to make decision when driving unmanned vehicle. The current surrounding environment of vehicle and the priori knowledge can make intelligent vehicle more intelligent while driving.

\section{CONFLICT OF INTEREST}

The authors confirm that this article content has no conflict of interest.

\section{ACKNOWLEDGEMENTS}

This paper is associated with the project of "Construction of Innovative Teams and Teacher Development for Universities and Colleges Under Beijing Municipality" and "The Importation and Development of High-Caliber Talents Project of Beijing Municipal Institutions" (CIT\&TCD2013 04074/IDHT20130513).

\section{REFERENCES}

[1] J. Hongyu and W. Ranghui, "Technology and the application of Global navigation and positioning system GNSS", GNSS world of China, 2010

[2] L. Yue and Q. Zhihe, "Navigation and positioning", Bei Jing: National Defence Industry Press, 2008.

[3] C. Po, "Simulation of INS/CNS integrated navigation system", Zheng Zhou: The PLA Information Engineering University, 2009.

[4] G.T. Schmidt, "INS/GPS Technology Trends", The Research and Technology Organisation of North Atlantic Treaty Organisation, 2011.

[5] D. Iwo and F. Chung, "Fuzzy Adaptive Unscented Kalman Filter for Ultra-Tight GPS/INSIntegration", International Symposium on Computational Intelligence and Design, pp. 229-235, 2010.

[6] A.S. Abbott and W.E. Lillo, "Global positioning system and inertial measuring unit unltratight coupling method", US, No.6516021, 2003. 
[7] D. Gebre-Egzibher,"What is the difference between 'loose','tight','ultra-tight' and 'deep' integration strategies for INS and GNSS? ", Inside GNSS, 2007

[8] L. Yinong, Y. Liu, Z. Ling and L. Shaobo. "Vehicle longitudinal and lateral coupling control based on sliding mode control", China mechanical engineering, vol 18, no. 7, 2007
[9] L. Mingbo, L. Jianchao and W. Chan. "UTM projection deformation processing", Northwest hydropower, 2010

[10] L. Yisong, W. Ning and S. Yamin. "Path planning algorithm based on grid method for virtual human", Computer Engineering \& Design, vol. 29, no. 5, pp. 1229-1231, 2008.

Received: September 16, 2014

Revised: December 23, 2014

Accepted: December 31, 2014

(C) Jin et al.; Licensee Bentham Open.

This is an open access article licensed under the terms of the Creative Commons Attribution Non-Commercial License (http://creativecommons.org/licenses/by-nc/4.0/) which permits unrestricted, non-commercial use, distribution and reproduction in any medium, provided the work is properly cited. 